\title{
Erratum to: Clinicopathological and genetic features of Chinese hereditary nonpolyposis colorectal cancer (HNPCC)
}

\author{
Fangqi Liu $\cdot$ Li Yang $\cdot$ Xiaoyan Zhou \\ Weiqi Sheng $\cdot$ Sanjun Cai $\cdot$ Lei Liu • \\ Peng Nan $\cdot$ Ye Xu
}

Published online: 4 December 2014

(c) Springer Science+Business Media New York 2014

Erratum to: Med Oncol (2014) 31:223

DOI 10.1007/s12032-014-0223-1

Unfortunately, Dr. Peng Nan was not indicated as co-corresponding author in the original publication. It has been corrected with this erratum.

The online version of the original article can be found under doi:10.1007/s12032-014-0223-1.

F. Liu $\cdot$ L. Yang $\cdot$ S. Cai $\cdot$ Y. Xu $(\bowtie)$

Department of Colorectal Surgery, Fudan University Shanghai

Cancer Center, Shanghai 200032, China

e-mail: xuye021@163.com; xu_shirley021@163.com

F. Liu $\cdot$ L. Yang $\cdot$ X. Zhou $\cdot$ W. Sheng $\cdot$ S. Cai $\cdot$ Y. Xu

Department of Oncology, Shanghai Medical College,

Fudan University, Shanghai 200032, China

X. Zhou - W. Sheng

Department of Pathology, Fudan University Shanghai Cancer

Center, Shanghai 200032, China

L. Liu

Shanghai Medical College of Fudan University,

Shanghai 200032, China

P. Nan $(\square)$

Ministry of Education Key Laboratory for Biodiversity Science

and Ecological Engineering, School of Life Sciences, Fudan

University, Shanghai 200433, China

e-mail: nanpeng@fudan.edu.cn 